\title{
Urgent warfarin reversal: know your options
}

\author{
Jacob Pendergrast, MD
}

Published online: 23 February 2010

(C) Canadian Anesthesiologists' Society 2010

In this issue of the Journal is a review by Grobler et al. on the management of patients taking vitamin $\mathrm{K}$ antagonists who require urgent surgical procedures. ${ }^{1}$ This topic is of interest to our readers and to much of the medical community; patients taking vitamin $\mathrm{K}$ antagonists (warfarin, for the most part) are all around us. Per capita use of warfarin in the United States quadrupled during the years 1988 to $2000,{ }^{2}$ and it tripled in Canada during the later interval of 1996 to $2006 .{ }^{3}$ In countries, such as Sweden and the United Kingdom, where such data have been published, it is estimated that $1 \%$ of the entire population is now taking warfarin. ${ }^{4}$ Much of this increase can be attributed to a series of trials conducted in the 1990s that demonstrated the value of warfarin in preventing cardioembolic disease in patients with atrial fibrillation. To a lesser extent, the growing prevalence of warfarin use is the result of a trend towards a longer duration of therapy for venous thromboembolic disease. As the incidence of both atrial fibrillation and venous thromboembolism increases exponentially among the elderly, the number of anticoagulated patients will continue to increase.

It has been established for some time that warfarin use can bring about significant reductions in mortality for patients with atrial fibrillation ${ }^{5}$ or venous thromboembolism. ${ }^{6}$ It is also true, however, that warfarin can be an exceptionally difficult medication to manage, with its narrow therapeutic index, myriad drug interactions, and susceptibility to changes in diet. Even within the strictures of a clinical trial, the risk of major bleeding while on

J. Pendergrast, MD ( $\square)$

Department of Laboratory Hematology, University Health

Network, Toronto General Hospital, 200 Elizabeth Street,

3 ES Room 306, Toronto, ON M5G 2C4, Canada

e-mail: jacob.pendergrast@uhn.on.ca warfarin ranges from 0.5 to 4.2 per 100 patients per year. Outside a clinical trial setting, the incidence may double. ${ }^{7}$ Despite these dangers, warfarin has dominated the field of outpatient thromboprophylaxis for over half a century and remains resistant to challenge by numerous contenders, such as vena cava filters, ${ }^{8}$ combined antiplatelet therapy, ${ }^{9}$ and low molecular weight heparin. ${ }^{10}$ Perhaps recently developed oral agents that possess more predictable dosing requirements will ultimately displace warfarin as the world's most popular anticoagulant. ${ }^{11}$ However, it is likely these agents will be considerably more expensive than warfarin and, more importantly, they currently lack an effective antidote, the quality that provides warfarin its singular advantage over all other alternatives.

Naturally, the antidote to vitamin $\mathrm{K}$ antagonists is vitamin $\mathrm{K}$. When given in adequate doses, vitamin $\mathrm{K}$ will completely and permanently reverse a warfarin-induced coagulopathy. However, even when administered intravenously, it may take four to six hours before the clinical effect of vitamin $\mathrm{K}$ becomes apparent, which is too long a wait for patients experiencing life- or limb-threatening bleeding or requiring emergent surgery. In these settings, it is necessary to supplement vitamin $\mathrm{K}$ with one of the following three bypassing agents: plasma, prothrombin complex concentrate (PCC), and recombinant activated factor seven (rVIIa). As Grobler et al. emphasize, none of these agents should be considered true antidotes to warfarin; their effect is lost within hours, and without the administration of vitamin $\mathrm{K}$, warfarin will continue to exert its anticoagulant effects for days after it has been discontinued.

Although the recently published American College of Chest Physicians' guidelines ${ }^{12}$ consider plasma, PCC, and rVIIa to be reasonable options for patients experiencing life-threatening bleeding from vitamin $\mathrm{K}$ antagonists, 
Grobler et al. rightly dismiss rVIIa as a serious candidate. In addition to its high cost and weak evidence base in this context, the half-life of rVIIa $(2.8 \mathrm{hr})$ is considerably shorter than the time it takes for vitamin $\mathrm{K}$ to act. To be precise, repeated dosing is likely to be required. Also, since rVIIa acts by over-riding rather than by restoring the body's normal coagulation pathways, it is arguably a cruder choice of bypassing agent than either plasma or PCC. Grobler et al. are supported in this position by other authorities who consider rVIIa to be a third-line agent, at best, for the emergency reversal of vitamin $\mathrm{K}$ antagonists. ${ }^{13-15}$

This leaves plasma and PCC. Plasma is the traditional choice and was effectively the only option in this country until Canadian Blood Services began supplying hospitals with Octaplex ${ }^{\circledR}$ in the spring of 2008. Like other PCC products, Octaplex ${ }^{\circledR}$ compares favourably with plasma regarding what it does not contain, most notably free water. Although the most recently published Canadian guidelines on plasma use recommends $5-8 \mathrm{~mL} \cdot \mathrm{kg}^{-1}$ for urgent warfarin reversal, ${ }^{16}$ it is likely that doses more than twice that (e.g., at least 3-4 units for an average-sized adult) will be necessary to correct an elevated international normalized ratio (INR). ${ }^{17}$ This may entail the infusion of up to $1 \mathrm{~L}$ of colloid, a volume that many patients would not be able to tolerate. Moreover, it takes time to infuse a therapeutic dose of plasma, a delay further compounded by the time clinicians must wait for its preparation, as typically it takes 20-30 min for frozen plasma to be thawed using standard waterbaths. For patients in extremis, these delays may be life-threatening. In addition to large volumes of free water, plasma contains many other unwanted substances that frequently are implicated in both minor and major transfusion reactions: immunoglobulins, cytokines, biologically active lipids, and citrate. Prothrombin complex concentrate products are largely free of such contaminants and, importantly, do not appear to cause transfusion-related acute lung injury, which is currently the leading cause of transfusion-related fatalities. Finally, PCCs have the advantage of convenience, particularly for the blood transfusion service: they are easy to store, easy to issue, and do not require pre-transfusion compatibility testing.

However, it is also the case that PCCs carry their own risks, and the concern regarding these risks, rightly or wrongly, has made many physicians hesitant to adopt the use of PCCs, particularly in North America. One concern is the risk of disease transmission. As with all fractionated plasma products, each dose of PCC exposes the patient to tens of thousands of blood donors, which historically has made coagulation factor concentrates efficient transmitters of human disease. However, the incorporation of dedicated pathogen reduction and inactivation processes by the plasma fractionation industry has dramatically reduced this risk, particularly regarding lipid-enveloped viruses. Despite the increased number of donor exposures, current modelling suggests that the infectious risk from an average vial of pathogen-inactivated plasma derivative is significantly lower than that posed from a single unit of a labile blood component like frozen plasma. ${ }^{18}$ Experience to date suggests that this assumption also holds true for prion diseases, which have been transmitted on four occasions by nonleukoreduced red blood cell transfusions, but only once by a plasma derivative. ${ }^{19}$

A second concern is that PCCs may induce thrombosis. Initially, PCCs were used primarily as a source of concentrated factor (F)IX for patients with hemophilia B. Even in this population of very coagulopathic patients, there were multiple reports in the 1970s of thrombotic complications following PCC administration. For patients taking vitamin $\mathrm{K}$ antagonists (who were, by definition, already at heightened risk of thrombosis), plasma transfusions seemed the wiser choice. Following a 1977 National Institutes of Health/Food and Drug Administration conference on the thrombogenicity of PCCs, the number of reported cases of PCC-related thrombosis declined dramatically, but market withdrawals of specific products due to association with fatal thrombosis continued into the 1990 s. $^{20}$ The latest generation of PCCs, which are manufactured according to European quality standards first published in 1996, hopefully have widened the therapeutic index of these products. ${ }^{21}$ Processes that can decrease the risk of thrombosis from PCCs include:

1) the addition of small amounts of heparin and/or antithrombin to prevent the in vitro autoconversion of the zymogen form of coagulation factors into their more potent activated form;

2) the maintenance of constituent coagulation factors (II, IX, $\mathrm{X}$, and/or VII) in careful proportion against one another;

3) the addition of natural anticoagulants of which patients on warfarin will also have deficiencies (e.g., proteins C and $\mathrm{S}$ );

4) The avoidance of PCCs in patients with coagulopathies from causes other than vitamin $\mathrm{K}$ deficiency, particularly those who may be accompanied by a concomitant state of hypercoagulability, such as disseminated intravascular coagulation;

5) careful dose adjustment based on patient weight and degree of baseline anticoagulation; and

6) use of slow infusion rates.

Octapharma, the manufacturer of Octaplex $\AA$, has incorporated all of the above considerations into its product monograph. As a result, the risk of thrombosis from this product appears to be very low when used according to 
manufacturer's recommendations. Unfortunately, the last two precautionary steps may so inconvenience clinicians that plasma may appear, on balance, to be an easier product to work with. In an attempt to simplify dose calculations, the Canadian National Advisory Committee on Blood Utilization has recommended a standardized dose of 1000 FIX units (generally equivalent to two vials of Octaplex) for adult patients weighing $50-90 \mathrm{~kg}$ and with a baseline INR from 1.5 to 4.0. However, the slow infusion rates recommended by the manufacturer may be an even greater source of irritation than dose calculation. When administered at the recommended maximal rate of $2-3 \mathrm{~mL} \cdot \mathrm{min}^{-1}$, it takes from 15 to $20 \mathrm{~min}$ to inject two vials of reconstituted Octaplex ${ }^{\circledR}$. Transferring the administered product into a mini-bag and infusing either by gravity or by volumetric pump allows the clinician to leave the patient's bedside and is an attractive alternative to the manufacturer's recommendations that the product be given by slow intravenous push. However, there are concerns that minibag infusion may entail some degree of product loss, either by adhering to the mini-bag or the infusion tubing or by product dilution. ${ }^{22}$ Hospitals that choose to adopt the minibag technique of Octaplex ${ }^{\circledR}$ infusion may find that higher doses are required than those recommended by the National Advisory Committee. Alternatively, observational studies of similar PCC products suggest that infusion rates two to three times faster than that recommended for Octaplex ${ }^{\circledR}$ may be safe. ${ }^{23}$ Either way, it is important that patients receiving PCCs be monitored carefully for signs of thrombotic complications and have a post-infusion INR performed immediately to confirm that an adequate dose was given.

In their review article, Grobler et al. appear to favour the use of Octaplex ${ }^{\circledR}$ over plasma for urgent warfarin reversal. Given our current knowledge about these products, are we ready to declare Octaplex ${ }^{\circledR}$ first-line therapy in this setting? This is not an easy question to answer, but the response is probably no, at least not yet. Unfortunately, there is not a strong evidence base for either product, and the few studies where they have had a head-to-head comparison were not adequately powered to detect differences in clinical outcomes. As Grobler et al. point out in their review, the relative rapidity by which a patient's INR is corrected cannot be assumed to correlate with improved patient outcome. Animal models comparing PCC and rVIIa, for example, showed an equivalent correction of coagulation test times that did not translate into an equivalent decrease in bleeding. A similar disjunction was observed in a large randomized placebo-controlled trial of vitamin $\mathrm{K}$ for the correction of warfarin overdose (INR 4.5-10.0). Vitamin $\mathrm{K}$ shortened coagulation times faster than placebo but did not decrease the risk of bleeding. ${ }^{24}$ Even in cases of warfarin-induced intra-cranial hemorrhage, the clinical significance of the patient's pretreatment INR and the rapidity with which it is corrected is unclear. ${ }^{25}$

Moreover, it should not be forgotten that hemostatic control is itself only a surrogate marker for clinically meaningful outcomes, such as patient morbidity and death, particularly when evaluating a therapeutic intervention that carries its own risks. The recently published study, Blood Conservation Using Antifibrinolytics in a Randomized Trial (BART), is but one example; the results of the study, which compared aprotinin with tranexamic acid post-cardiopulmonary bypass, showed aprotinin decreased bleeding but increased mortality. ${ }^{26}$ Experiences to date with the offlabel use of rVIIa has been similar. In patients with spontaneous intracerebral hemorrhage (ICH), rVIIa decreased hematoma growth compared with placebo but without net benefit in the clinically-meaningful outcomes of death or disability, perhaps reflecting the increased risk of arterial thrombotic events that rVIIa provoked. ${ }^{27}$

Given the above uncertainties, perhaps clinicians are best advised to select either Octaplex ${ }^{\circledR}$ or plasma based on the particular vulnerabilities of the individual patient. Collaborative discussion with a hematologist or the blood bank medical director is often very helpful in this regard. For patients who require emergent correction of a warfarininduced coagulopathy, who are at risk of volume overload, or who have a history of adverse transfusion reactions, Octaplex ${ }^{\circledR}$ is probably the preferred choice. A plasma transfusion may be more prudent for patients at high risk of thrombosis, with a history of heparin-induced thrombocytopenia, or whose coagulopathy may be exacerbated by other causes, such as disseminated intravascular coagulation. Canadian hospitals that serve patients who are taking vitamin $\mathrm{K}$ antagonists (i.e., most hospitals) would be advised to stock plasma, Octaplex ${ }^{\circledR}$, or both. The use of either product should be guided by institutional guidelines and reviewed regularly, ideally by a Hospital Transfusion Committee. In addition, given the relative scarcity of Octaplex ${ }^{\circledR}$ in Canada, smaller hospitals should probably refrain from stocking this product until they have established a redistribution network whereby larger hospitals receive unused vials as they near the expiry date.

Ultimately, we will not be certain whether plasma or Octaplex ${ }^{\circledR}$ is preferred for correction of warfarin coagulopathy until the two are compared in a well-designed randomized controlled trial using meaningful clinical outcomes, such as death, disability, or need for hospitalization. Such a trial would need to be substantially larger than the trials that have thus far been performed for PCCs, but enrolment should not pose too much of a challenge. As noted, patients taking warfarin constitute a large and growing 
population, and many will require an urgent surgical procedure at some point in their lives.

\section{Neutralisation urgente de la warfarine: soyez informés de vos options!}

Dans ce numéro du Journal se trouve un compte-rendu de Grobler et coll. portant sur la prise en charge de patients traités avec des antagonistes de la vitamine $\mathrm{K}$ et nécessitant des interventions chirurgicales urgentes. ${ }^{1}$ Nos lecteurs et la communauté médicale seront intéressés par un tel sujet; en effet, nous sommes entourés de patients prenant des antagonistes de la vitamine $\mathrm{K}$ (principalement la warfarine). L'utilisation par personne de warfarine aux États-Unis a quadruplé entre 1988 et $2000,{ }^{2}$ et elle a triplé au Canada entre 1996 et 2006. ${ }^{3}$ Dans d'autres pays tels que la Suède et le Royaume Uni, pour lesquels de telles données ont été publiées, on estime qu'un pour cent de la population globale prend maintenant de la warfarine. ${ }^{4}$ Cette augmentation peut en grande partie s'expliquer par une série d'études menées au cours des années 1990 et démontrant le rôle positif de la warfarine dans la prévention des maladies cardio-emboliques chez les patients souffrant de fibrillation auriculaire. Dans une moindre mesure, l'utilisation de plus en plus répandue de la warfarine découle d'une tendance vers une durée prolongée du traitement pour soigner les maladies thromboemboliques veineuses. L'incidence de fibrillation auriculaire et de thromboembolie veineuse augmentant exponentiellement chez les personnes âgées, le nombre de patients sous traitement anticoagulant continuera lui aussi d'augmenter.

Nous savons depuis un certain temps que la warfarine peut réduire significativement l'incidence de mortalité chez les patients souffrant de fibrillation auriculaire ${ }^{5}$ ou de thromboembolie veineuse. ${ }^{6}$ Toutefois, nous savons aussi que la warfarine peut être un médicament particulièrement difficile à administrer et à gérer, étant donné son indice thérapeutique étroit, ses nombreuses interactions médicamenteuses et sa sensibilité à toute modification dans l'alimentation du patient. Même dans le cadre rigoureusement contrôlé d'une étude clinique, le risque de saignement majeur pendant un traitement à la warfarine oscille entre 0,5 et 4,2 par 100 patients par année. Hors du cadre d'une étude clinique, cette incidence pourrait doubler. ${ }^{7}$ En dépit de ces dangers, la warfarine est à la tête des thromboprophylaxies ambulatoires depuis plus d'un demi-siècle et résiste à la concurrence de plusieurs autres candidats, notamment les filtres de veine cave, ${ }^{8}$ les traitements antiplaquettaires combinés ${ }^{9}$ et l'héparine à bas poids moléculaire. ${ }^{10}$ Peut-être que les agents oraux récemment mis au point, qui ont des posologies plus prévisibles, remplaceront en fin de compte la warfarine en tant qu'anticoagulant le plus populaire dans le monde. ${ }^{11}$ Cependant, ces agents seront probablement bien plus coûteux que la warfarine, mais surtout, il se peut qu'ils n'aient pas d'antidote efficace-ce qui donne à la warfarine son avantage unique par rapport à toutes les autres alternatives.

L'antidote aux antagonistes de la vitamine $\mathrm{K}$ est, on s'en doute, la vitamine $\mathrm{K}$. Si elle est administrée à la bonne dose, la vitamine $\mathrm{K}$ renversera complètement et de façon permanente une coagulopathie induite par la warfarine. Par contre, même lorsqu'elle est administrée par voie intraveineuse, la vitamine $\mathrm{K}$ peut prendre quatre à six heures avant d'avoir un effet cliniquement apparent, ce qui est bien trop long pour les patients dont les saignements sont potentiellement fatals ou compromettent un membre, ou ceux qui nécessitent une chirurgie urgente. Dans de telles situations, il faut ajouter à la vitamine $\mathrm{K}$ l'un des trois agents de contournement suivants : du plasma, du concentré de complexe prothrombique (CCP) ou du facteur sept recombinant activé (rVIIa). Comme le soulignent Grobler $e t$ coll., aucun de ces agents ne devrait être considéré comme un véritable antidote à la warfarine; leur effet n'est que temporaire et, sans l'administration de vitamine $\mathrm{K}$, la warfarine continuera d'exercer ses effets anticoagulants pendant plusieurs jours après son interruption.

Bien que les directives récemment publiées par le American College of Chest Physicians décrivent le plasma, le CCP et le rVIIa comme des alternatives raisonnables à administrer aux patients subissant des saignements potentiellement fatals causés par les antagonistes de la vitamine K, Grobler et coll. écartent le rVIIa de la liste des candidats de façon tout à fait justifiée. Outre son coût élevé et le peu de données probantes soutenant son effet dans un tel contexte, la demi-vie du rVIIa $(2,8 \mathrm{~h})$ est considérablement plus courte que le temps qu'il faut pour que la vitamine $\mathrm{K}$ agisse - en fait, il est probable que des doses répétées soient nécessaires. De plus, étant donné que le rVIIa agit en supplantant les voies de coagulation normales du corps plutôt qu'en les restaurant, il constitue, selon toutes probabilités, une option plus grossière d'agent de contournement que le plasma ou le CCP. Grobler et coll. ne sont pas les seuls à être de cet avis. En effet, d'autres autorités considèrent le rVIIa au mieux comme un agent de troisième ligne pour la neutralisation d'urgence des antagonistes de la vitamine $\mathrm{K} .{ }^{13-15}$

Restent donc le plasma et le CCP. Le choix habituel est le plasma, qui était en fait la seule option au Canada jusqu'à ce que la Société canadienne du sang commence à fournir de l'Octaplex ${ }^{\circledR}$ aux hôpitaux au printemps 2008 . Tout comme les autres produits de CCP, l'Octaplex ${ }^{\circledR}$ soutient favorablement la comparaison avec le plasma 
quant à ce qu'il ne contient pas, et tout particulièrement de l'eau libre. Bien que les directives canadiennes les plus récentes concernant l'utilisation de plasma préconisent un dosage de $5-8 \mathrm{~mL} \cdot \mathrm{kg}^{-1} \mathrm{~s}$ 'il faut réaliser une neutralisation urgente de la warfarine ${ }^{16}$ il est probable que des doses au moins deux fois plus élevées (par ex., 3-4 unités pour un adulte de taille moyenne) soient nécessaires pour corriger un rapport international normalisé (INR) élevé. ${ }^{17}$ Ceci pourrait impliquer la perfusion d'un maximum d'1 L de colloïdes, un volume que nombre de patients ne pourraient tolérer. En outre, la perfusion d'une dose thérapeutique de plasma prend du temps, un délai encore aggravé par le temps qu'il faut à sa préparation; en effet, il faut en général compter 20-30 min pour que le plasma congelé soit décongelé au bain-marie. Pour des patients en situation extrême, de tels délais pourraient s'avérer fatals. Outre d'importants volumes d'eau libre, le plasma contient d'autres substances indésirables qui provoquent souvent des réactions à la transfusion, qu'elles soient mineures ou majeures : les immunoglobulines, les cytokines, les lipides biologiquement actifs et le citrate. Les produits de concentré de complexe prothrombique ne contiennent presque pas de tels agents contaminateurs, mais surtout ils ne semblent pas causer de lésion pulmonaire aiguë due à la transfusion, ce qui est actuellement la principale cause de décès liés à la transfusion. Enfin, les CCP sont pratiques, particulièrement pour le service de transfusion sanguine : ils sont faciles à entreposer, faciles à distribuer, et ne nécessitent pas de test de compatibilité avant d'être transfusés.

Toutefois, les CCP possèdent également leur lot de risques, et l'inquiétude face à ces risques est ce qui, de façon justifiée ou non, fait que nombre de médecins hésitent à les utiliser, tout particulièrement en Amérique du Nord. L'une des inquiétudes touche au risque de transmission de maladies. Tout comme c'est le cas avec tous les produits plasmatiques fractionnés, chaque dose de CCP expose le patient qui la reçoit à des dizaines de milliers de donneurs de sang, ce qui, d'un point de vue historique, a fait des concentrés de facteurs de coagulation d'excellents transmetteurs de maladies humaines. Le secteur du fractionnement plasmatique a toutefois mis en place des processus spécifiques pour réduire et inactiver les pathogènes, ce qui a considérablement réduit ce risque, en particulier au niveau des virus enveloppés de lipides. En dépit du nombre accru de donneurs auquel le patient est ainsi exposé, les modèles actuels suggèrent que le risque d'infection à partir d'un flacon de dérivé plasmatique dans lequel les pathogènes ont été inactivés est, en moyenne, significativement plus bas que le risque encouru par le patient lorsqu'il est exposé à une seule unité de composé sanguin labile tel que le plasma congelé. ${ }^{18}$ À ce jour, l'expérience suggère que cette hypothèse s'applique également aux maladies à prions, dont quatre cas ont été rapportés à la suite de transfusions d'érythrocytes leucoréduits, mais un seul avec un dérivé plasmatique. ${ }^{19}$

Une deuxième inquiétude porte sur le fait que les CCP pourraient provoquer des thromboses. À l'origine, les CCP étaient principalement utilisés en tant que source du facteur concentré (F)IX pour les patients atteints d'hémophilie B. Même dans cette population de patients très à risque de coagulopathie, de nombreuses complications thrombotiques ont été rapportées dans les années 1970 après administration de CCP. Pour les patients prenant des antagonistes de la vitamine $\mathrm{K}$ (qui, par définition, couraient déjà un risque plus élevé de thrombose), les transfusions de plasma semblaient constituer la meilleure option. En 1977, à la suite d'une conférence conjointe des National Institutes of Health et de la Food and Drug Administration américains portant sur la thrombogénicité des CCP, le nombre de cas rapportés de thromboses associées aux CCP a considérablement diminué, mais les retraits commerciaux de produits spécifiques en raison d'une association avec des cas fatals de thrombose ont continué jusque dans les années $1990 .^{20}$ La génération la plus récente de CCP, fabriqués conformément aux normes de qualité européennes publiées pour la première fois en 1996, auront, avec un peu de chance, étendu quelque peu l'indice thérapeutique de ces produits. ${ }^{21}$ Parmi les processus à même de réduire le risque de thrombose liée aux CCP, citons :

7) l'ajout de petites quantités d'héparine et/ou d'antithrombine afin de prévenir l'autoconversion in vitro de la forme zymogène des facteurs de coagulation en leur forme activée la plus puissante;

8) Le maintien d'une proportion précise de facteurs de coagulation constitutifs (II, IX, X, et/ou VII) l'un par rapport à l'autre;

9) l'ajout d'anticoagulants naturels dont les patients sous warfarine ont besoin en raison de carences (par ex., protéines $\mathrm{C}$ et $\mathrm{S}$ );

10) le non-recours à des $\mathrm{CCP}$ chez les patients souffrant de coagulopathies provoquées par des causes autres qu'une carence en vitamine $\mathrm{K}$, en particulier chez ceux qui pourraient souffrir d'un état conjoint d'hypercoagulabilité, comme par ex. une coagulation intravasculaire disséminée;

11) un ajustement précis des doses selon le poids du patient et son degré initial d'anticoagulation; et

12) le recours à des débits de perfusion lents.

Octapharma, le fabricant d'Octaplex ${ }^{\circledR}$, a tenu compte de toutes ces considérations dans sa monographie du produit. Par conséquent, le risque de thrombose provoqué par ce produit semble très bas lorsqu'il est utilisé conformément aux recommandations du fabricant. Malheureusement, les deux dernières précautions à respecter pourraient incommoder les cliniciens à tel point que le plasma 
pourrait sembler, tout bien considéré, être un produit avec lequel il est plus facile de travailler. Dans le but de simplifier le calcul des doses à administrer, le Comité consultatif national sur l'utilisation des produits sanguins du Canada a recommandé une dose standard de 1000 unités FIX (en général équivalente à deux flacons d'Octaplex) pour les patients adultes d'un poids de 50 à $90 \mathrm{~kg}$ et avec un INR de base de 1,5 à 4,0. Les débits de perfusion lents recommandés par le fabricant pourraient toutefois constituer une source encore plus importante d'exaspération que le calcul des doses. Lors d'une administration au débit maximal recommandé de $2-3 \mathrm{~mL} \cdot \mathrm{min}^{-1}$, il faut de 15 à 20 min pour injecter deux flacons d'Octaplex ${ }^{\circledR}$ reconstitué. Le transfert du produit administré dans un minisac et sa perfusion par gravité ou par pompe volumétrique permettent au clinicien de quitter le chevet du patient, ce qui constitue une alternative séduisante aux recommandations du fabricant, selon lesquelles le produit devrait être administré par perfusion intraveineuse lente. Cependant, l'inquiétude ici est qu'une perfusion par minisac entraîne une certaine perte du produit, soit par adhérence au minisac ou à la tubulure de perfusion, soit par dilution du produit. ${ }^{22}$ Dès lors, il se peut que les hôpitaux choisissant d'adopter la technique de perfusion de l'Octaplex ${ }^{\circledR}$ par minisac découvrent qu'une dose plus élevée que celle recommandée par le Comité consultatif national soit nécessaire. En revanche, des études observationnelles portant sur des produits de CCP similaires suggèrent que des débits de perfusion deux à trois fois plus rapides que ceux recommandés pour l'Octaplex ${ }^{\circledR}$ pourraient être sécuritaires. ${ }^{23}$ Quoi qu'il en soit, il est important de surveiller de près les patients recevant des CCP pour dépister les signes de complications thrombotiques et de mesurer l'INR du patient immédiatement après la perfusion afin de confirmer qu'une dose appropriée a été administrée.

Dans leur compte-rendu, Grobler et coll. semblent être en faveur de l'utilisation d'Octaplex ${ }^{\circledR}$ plutôt que de plasma lorsque la neutralisation de la warfarine est urgente. Étant donné nos connaissances actuelles concernant ces produits, sommes-nous prêts à faire de l'Octaplex ${ }^{\circledR}$ notre traitement de première ligne dans un tel contexte? Il s'agit là d'une question épineuse, mais la réponse est probablement non-du moins pour le moment. Malheureusement, nous ne disposons pas de données probantes solides soutenant un produit ou l'autre, et la puissance des quelques études les comparant ne suffit pas pour détecter des différences au niveau des devenirs cliniques. Comme Grobler et coll. le mentionnent, on ne peut prendre pour acquis que la rapidité relative avec laquelle l'INR d'un patient est corrigé est corrélée à un meilleur devenir du patient. Les modèles animaux comparant les CCP et le rVIIa, par exemple, ont montré une correction équivalente dans les temps de test de coagulation qui ne s'est pas traduite par une réduction équivalente du saignement. Une discordance similaire a été observée dans une vaste étude randomisée et contrôlée par placebo examinant l'utilisation de vitamine $\mathrm{K}$ pour corriger une surdose de warfarine (INR 4,5-10,0). La vitamine K a réduit les temps de coagulation plus rapidement que le placebo mais n'a pas réduit le risque de saignement. ${ }^{24}$ Même dans les cas d'hémorragie intracrânienne provoquée par warfarine, l'importance clinique de l'INR du patient avant le traitement et la rapidité à laquelle il est corrigé demeurent peu claires. ${ }^{25}$

En outre, il ne faut pas oublier que le contrôle de l'hémostase n'est en soi qu'un marqueur de substitution des devenirs cliniquement significatifs tels que la morbidité et le décès, en particulier lors de l'évaluation d'une intervention thérapeutique qui entraîne ses propres risques. L'étude BART (Blood Conservation Using Antifibrinolytics in a Randomized Trial), publiée récemment, n'est qu'un exemple; les résultats de cette étude, qui a comparé l'aprotinine à l'acide tranexamique après la circulation extracorporelle, ont montré que l'aprotinine réduisait le saignement mais augmentait la mortalité. ${ }^{26}$ Les expériences à notre disposition à ce jour concernant l'utilisation non conforme de rVIIa ont donné des résultats semblables. Chez les patients souffrant d'une hémorragie intracérébrale (HIC) spontanée, le rVIIa a réduit la croissance de l'hématome par rapport au placebo mais sans entraîner d'avantage clair au niveau des devenirs cliniquement significatifs, soit le décès ou l'invalidité. Ces résultats reflètent peut-être le risque accru de complications thrombotiques artérielles provoquées par le rVIIa. ${ }^{27}$

Étant donné les incertitudes exprimées plus haut, le meilleur conseil à donner aux cliniciens est peut-être qu'ils choisissent l'Octaplex ${ }^{\circledR}$ ou le plasma selon les vulnérabilités particulières de chaque patient. Des discussions en collaboration avec un hématologue ou le directeur médical de la banque de sang sont souvent très utiles à cet égard. Pour les patients nécessitant une correction immédiate d'une coagulopathie provoquée par la warfarine, qui présentent un risque de surcharge volumique ou qui ont des antécédents de réactions secondaires à la transfusion, l'Octaplex ${ }^{\circledR}$ est probablement à privilégier. Une transfusion de plasma pourrait en revanche être la voie de la prudence pour les patients présentant un risque élevé de thrombose, avec des antécédents de thrombocytopénie induite par l'héparine, ou dont la coagulopathie pourrait être exacerbée par d'autres causes telles qu'une coagulation intravasculaire disséminée. Les hôpitaux canadiens au service de patients prenant des antagonistes de la vitamine $\mathrm{K}$ (en d'autres termes, la plupart des hôpitaux) devraient faire des réserves de plasma, d'Octaplex ${ }^{\circledR}$ ou des deux produits. L'utilisation de l'un et l'autre produit devrait se fonder sur des directives institutionnelles et révisées régulièrement, idéalement par le comité de transfusion de l'hôpital. En outre, étant donné la 
rareté relative de l'Octaplex ${ }^{\circledR}$ au Canada, les hôpitaux de moindre envergure devraient peut-être ne pas faire de réserves de ce produit avant d'avoir mis sur pied un réseau de redistribution par le biais duquel les hôpitaux plus importants reçoivent les flacons non utilisés lorsque leur date de péremption approche.

En fin de compte, nous ne saurons pas s'il faut préférer le plasma ou l'Octaplex ${ }^{\circledR}$ pour la correction d'une coagulopathie induite par warfarine avant que ces deux produits ne soient comparés dans une étude randomisée contrôlée bien conçue et examinant des devenirs cliniques significatifs comme le décès, l'invalidité ou le besoin d'hospitalisation. Une telle étude devrait être considérablement plus vaste que les études qui ont été réalisées pour les $\mathrm{CCP}$ à ce jour, mais le recrutement ne devrait pas poser de problème. Comme nous l'avons mentionné, les patients sous warfarine sont une population importante et croissante, et nombre d'entre eux nécessiteront une intervention chirurgicale urgente à un moment donné de leurs vies.

Competing interests None declared.

\section{References}

1. Grobler C, Callum J, McCluskey SA, Reversal of vitamin K antagonists prior to urgent surgery. Can J Anesth 2010; 57: DOI: 10.1007/s12630-009-9250-3.

2. Flaherty $M L$, Kissela B, Woo D, et al. The increasing incidence of anticoagulant-associated intracerebral hemorrhage. Neurology 2007; 68: 116-21.

3. Jackevicius CA, Cox JL, Carreon D, et al. Canadian Cardiovascular Outcomes Research Team. Long-term trends in use of and expenditures for cardiovascular medications in Canada. CMAJ 2009; 181: E19-28.

4. Kim MJ, Huang SM, Meyer UA, Rahman A, Lesko LJ. A regulatory science perspective on warfarin therapy: a pharmacogenetic opportunity. J Clin Pharmacol 2009; 49: 138-46.

5. Aguilar MI, Hart R, Oral anticoagulants for preventing stroke in patients with non-valvular atrial fibrillation and no previous history of stroke or transient ischemic attacks. Cochrane Database Syst Rev 2005; (3): CD001927.

6. Barritt DW, Jordan SC. Anticoagulant drugs in the treatment of pulmonary embolism, A controlled trial. Lancet 1960; 1: 1309-12.

7. Schulman S. Clinical practice. Care of patients receiving longterm anticoagulant therapy. N Engl J Med 2003; 349: 675-83.

8. PREPIC Study Group. Eight-year follow-up of patients with permanent vena cava filters in the prevention of pulmonary embolism: the PREPIC (Prevention du Risque d'Embolie Pulmonaire par Interruption Cave) randomized study. Circulation 2005; 112: 416-22.

9. ACTIVE Writing Group of the ACTIVE Investigators, Connolly $S$, Pogue J, Hart R, et al. Clopidogrel plus aspirin versus oral anticoagulation for atrial fibrillation in the Atrial Fibrillation
Clopidogrel Trial with Irbesartan for Prevention of Vascular Events (ACTIVE W): a randomised controlled trial. Lancet 2006; 367: 1903-12.

10. van der Heijden JF, Hutten BA, Buller HR, Prins $M H$, Vitamin $\mathrm{K}$ antagonists or low-molecular weight heparin for the long term treatment of symptomatic venous thromboembolism. Cochrane Database Syst Rev 2001; (1): CD002001.

11. Garcia D, Libby E, Crowther MA. The new oral anticoagulants. Blood 2010; 115: 15-20.

12. Ansell J, Hirsh J, Hylek E, et al. Pharmacology and management of the vitamin $\mathrm{K}$ antagonists: American College of Chest Physicians Evidence-Based Clinical Practice Guidelines (8th Edition). Chest 2008; 133(6 Suppl): 160S-98S.

13. Baglin TP, Keeling DM, Watson HG, British Committee for Standards in Haematology. Guidelines on oral anticoagulation (warfarin): third edition-2005 update. Br J Haematol 2006; 132: 277-85.

14. Schulman S, Bijsterveld NR. Anticoagulants and their reversal. Transfus Med Rev 2007; 21: 37-48.

15. Crowther MA, Warkentin TE. Bleeding risk and the management of bleeding complications in patients undergoing anticoagulant therapy: focus on new anticoagulant agents. Blood 2008; 111: 4871-9.

16. Expert Working Group. Guidelines for red blood cell and plasma transfusion for adults and children. CMAJ 1997; 156(11 suppl): S1-24.

17. Holland LL, Brooks JP. Toward rational fresh frozen plasma transfusion: the effect of plasma transfusion on coagulation test results. Am J Clin Pathol 2006; 126: 133-9.

18. Horowitz B, Busch M. Estimating the pathogen safety of manufactured human plasma products: application to fibrin sealants and to thrombin. Transfusion 2008; 48: 1739-53.

19. Ward HJ, MacKenzie JM, Llewelyn CA, et al. Variant Creutzfeldt-Jakob disease and exposure to fractionated plasma products. Vox Sang 2009; 97: 207-10.

20. Hellstern P, Halbmayer WM, Kohler M, Seitz R, Muller-Berghaus $G$. Prothrombin complex concentrates: indications, contraindications, and risks a task force summary. Thromb Res 1999; 95 (4 Suppl 1): S3-6.

21. European Pharmacopoeia Commission. Human prothrombin complex, freeze-dried. Pharmeuropa 1996; 8: 29-30.

22. McLeod AG, Walker IR, Zheng S, Hayward CP. Loss of factor VIII activity during storage in PVC containers due to adsorption. Haemophilia 2000; 6: 89-92.

23. Pabinger I, Tiede A, Kalina U, Knaub S, Germann R, Ostermann $H$; Beriplex ${ }^{\circledR}$ P/N Anticoagulation Reversal Study Group. Impact of infusion speed on the safety and effectiveness of prothrombin complex concentrate: a prospective clinical trial of emergency anticoagulation reversal. Ann Hematol 2009; DOI: 10.1007/ s00277-009-0830-7.

24. Crowther MA, Ageno W, Garcia D, et al. Oral vitamin $\mathrm{K}$ versus placebo to correct excessive anticoagulation in patients receiving warfarin: a randomized trial. Ann Intern Med 2009; 150: 293-300.

25. Zubkov AY, Mandrekar JN, Claassen DO, Manno EM, Wijdicks $E F$, Rabinstein AA. Predictors of outcome in warfarin-related intracerebral hemorrhage. Arch Neurol 2008; 65: 1320-5.

26. Fergusson DA, Hebert PC, Mazer CD, et al. BART Investigators. A comparison of aprotinin and lysine analogues in high-risk cardiac surgery. N Engl J Med 2008; 358: 2319-31.

27. Mayer SA, Brun NC, Begtrup K, et al. FAST Trial Investigators. Efficacy and safety of recombinant activated factor VII for acute intracerebral hemorrhage. N Engl J Med 2008; 358: 2127-37. 